\title{
SOME REMARKS ON ESSENTIALLY NORMAL SUBMODULES
}

\author{
RONALD G. DOUGLAS AND KAI WANG
}

\begin{abstract}
Given a $*$-homomorphism $\sigma: C(M) \rightarrow \mathscr{L}(\mathcal{H})$ on a Hilbert space $\mathcal{H}$ for a compact metric space $M$, a projection $P$ onto a subspace $\mathcal{P}$ in $\mathcal{H}$ is said to be essentially normal relative to $\sigma$ if $[\sigma(\varphi), P] \in \mathcal{K}$ for $\varphi \in C(M)$, where $\mathcal{K}$ is the ideal of compact operators on $\mathcal{H}$. In this note we consider two notions of span for essentially normal projections $P$ and $Q$, and investigate when they are also essentially normal. First, we show the representation theorem for two projections, and relate these results to Arveson's conjecture for the closure of homogenous polynomial ideals on the Drury-Arveson space. Finally, we consider the relation between the relative position of two essentially normal projections and the $K$ homology elements defined for them.
\end{abstract}

\section{INTRODUCTION}

Spurred by a question of Arveson [1, 2, 3, 4], several researchers have been considering when certain submodules of various Hilbert modules of holomorphic functions on the unit ball in $\mathbb{C}^{n}$ are essentially normal. In particular, Guo and the second author showed in [17] that the closure of a principal homogenous polynomial ideal in the DruryArveson space in $\mathbb{B}^{n}$ is essentially normal. More recently, the authors have shown in [13] that the closure of all principal polynomial ideals in the Bergman module on the unit ball are essentially normal. Other results have been obtained by Arveson [5], Douglas [10, 11], the first author and Sarkar [12], Eschmeier [14], Kennedy [19], and Shalit [20].

The Arveson conjecture concerns the closure of an arbitrary homogeneous polynomial ideal which, in general, is not singly generated. For the case of $n=1$, one knows that a pure hyponormal operator submodule is essentially normal if it is finitely generated. The basis on which this result depends is the Berger-Shaw Theorem [8].

For ideals that are not principal, or singly generated, the results in the several variable case are few. Guo in [15] firstly proved Arveson's conjecture in case of the dimension $n=2$. Guo and the second author established in [16, 17] essential normality when $n=3$ or the dimension of the zero variety of the homogeneous ideal is one or less, the opposite extreme, more or less, of the case of principal ideals. There is also a result of Shalit [20] which holds for ideals having a "very nice" basis relative to the norm. More recently, Kennedy [19] extended that result in another direction, considering when the linear span of the closures of polynomial ideals is closed. He gives some examples, but it would appear that not all non-principal ideals are covered by this

2010 Mathematics Subject Classification. 47A13, 46E22,46H25, 47A53.

Key words and phrases. Arveson conjecture, essentially normal submodules.

The second author was supported by NSFC, the Department of Mathematics at Texas A\&M University and Laboratory of Mathematics for Nonlinear Science at Fudan University. 
result. One should note that these results when the linear span of two essentially normal submodules is closed is implicit in the work of Arveson [5, Theorem 4.4].

In this note, we explore a more general version of the question of when the linear span of two essentially normal submodules is also essentially normal. We show that this result contains one aspect of the results of Shalit and Kennedy.

Our work does not depend on the special nature of the submodules; that is, we do not assume any connection with any underlying algebraic structure, only the fact that the linear span is closed.

There is more than one sense of the span of two submodules relevant in this context: the first is the obvious one defined to be the closure of the linear span of two submodules $\mathcal{P}$ and $\mathcal{Q}$, while the second one considers the span modulo the ideal of compact operators. If $P$ and $Q$ denote the orthogonal projections onto $\mathcal{P}$ and $\mathcal{Q}$, respectively, then we will show that this notion makes sense if 0 is an isolated point in the essential spectrum of $P+Q$.

We consider the first notion in Section 2, and the results obtained are based on the structure theorem for two projections. The latter notion of the essential span is taken up in Section 3. We apply these results to the context of Arveson's conjecture and raise some questions. In particular, we assume that there is a $*$-homomorphism $\sigma$ of $C(M)$ for some compact metric space $M$ and the projections essentially commute with the range of $\sigma$. Finally, in Section 4 we observe that an essentially normal projection determines an element of the odd $K$-homology group for some compact subset of $M$ and consider the relation of the K-homology elements defined by two essentially normal submodules and their sum.

\section{Refinement of the Two Projection Representation}

Our results in this section are based on refinements of the structure theorem for two projections [6, 18].

Let $P$ and $Q$ be two projections on the Hilbert space $\mathcal{H}$. Then there exist operators $S_{1}: \mathcal{P} \rightarrow \mathcal{P}, S_{2}: \mathcal{P}^{\perp} \rightarrow \mathcal{P}^{\perp}$ and $X: \mathcal{P}^{\perp} \rightarrow \mathcal{P}$, where $\mathcal{P}=$ ran $P$ with $0_{\mathcal{P}} \leq S_{1} \leq$ $I_{\mathcal{P}}, 0_{\mathcal{P} \perp} \leq S_{2} \leq I_{\mathcal{P} \perp}$ and $\|X\| \leq 1$, such that

$$
P=\left(\begin{array}{cc}
I_{\mathcal{P}} & 0 \\
0 & 0_{\mathcal{P}^{\perp}}
\end{array}\right) \text { and } Q=\left(\begin{array}{cc}
S_{1} & X \\
X^{*} & S_{2}
\end{array}\right)
$$

Moreover, if we set $\mathcal{P}=\mathcal{P}_{1} \oplus \mathcal{P}_{2} \oplus \mathcal{P}_{3}$ and $\mathcal{P}^{\perp}=\mathcal{Q}_{1} \oplus \mathcal{Q}_{2} \oplus \mathcal{Q}_{3}$, where $\mathcal{P}_{1}=\{x \in \mathcal{P}$ : $\left.S_{1} x=0\right\}, \mathcal{P}_{2}=\left\{x \in \mathcal{P}: S_{1} x=x\right\}, \mathcal{P}_{3}=\mathcal{P} \ominus\left(\mathcal{P}_{1} \oplus \mathcal{P}_{2}\right), \mathcal{Q}_{2}=\left\{x \in \mathcal{P}^{\perp}: S_{2} x=x\right\}$, $\mathcal{Q}_{3}=\left\{x \in \mathcal{P}^{\perp}: S_{2} x=0\right\}$, and $\mathcal{Q}_{1}=\mathcal{P}^{\perp} \ominus\left(\mathcal{Q}_{2} \oplus \mathcal{Q}_{3}\right)$, then we have

$$
\begin{aligned}
S_{1} & =\left(\begin{array}{ccc}
0_{\mathcal{P}_{1}} & 0 & 0 \\
0 & I_{\mathcal{P}_{2}} & 0 \\
0 & 0 & S_{1}^{\prime}
\end{array}\right) \in \mathscr{L}\left(\mathcal{P}_{1} \oplus \mathcal{P}_{2} \oplus \mathcal{P}_{3}\right) \text { with } S_{1}^{\prime} \in \mathscr{L}\left(\mathcal{P}_{3}\right), \\
S_{2} & =\left(\begin{array}{ccc}
S_{2}^{\prime} & 0 & 0 \\
0 & I_{\mathcal{Q}_{2}} & 0 \\
0 & 0 & 0_{\mathcal{Q}_{3}}
\end{array}\right) \in \mathscr{L}\left(\mathcal{Q}_{1} \oplus \mathcal{Q}_{2} \oplus \mathcal{Q}_{3}\right) \text { with } S_{2}^{\prime} \in \mathscr{L}\left(\mathcal{Q}_{1}\right), \text { and }
\end{aligned}
$$




$$
X=\left(\begin{array}{ccc}
0 & 0 & 0 \\
0 & 0 & 0 \\
X^{\prime} & 0 & 0
\end{array}\right) \in \mathscr{L}\left(\mathcal{Q}_{1} \oplus \mathcal{Q}_{2} \oplus \mathcal{Q}_{3}, \mathcal{P}_{1} \oplus \mathcal{P}_{2} \oplus \mathcal{P}_{3}\right) \text { with } X^{\prime} \in \mathscr{L}\left(\mathcal{Q}_{1}, \mathcal{P}_{3}\right)
$$

These results are all straightforward.

Further, using matrix computations and the fact that $Q^{2}=Q=Q^{*}$, one shows that there exists an isometry $V$ from $\mathcal{Q}_{1}$ onto $\mathcal{P}_{3}$ such that $V^{*} S_{1}^{\prime} V=I_{\mathcal{Q}_{1}}-S_{2}^{\prime}$. We refer the reader to [18] for a detailed argument. Therefore, we have derived the standard model for two projections.

Theorem 2.1. Two projections $P$ and $Q$ on a Hilbert space $\mathcal{H}$ are determined by

(1) a decomposition $\mathcal{H}=\mathcal{H}_{0} \oplus \mathcal{H}_{1} \oplus \mathcal{H}^{\prime} \oplus \mathcal{H}^{\prime} \oplus \mathcal{H}_{2} \oplus \mathcal{H}_{3}$, and

(2) a positive contraction $S \in \mathcal{L}\left(\mathcal{H}^{\prime}\right)$ with $\{0,1\}$ not in its point spectrum.

In this case, one has

$$
P=\left(\begin{array}{cccccc}
I_{\mathcal{H}_{0}} & 0 & 0 & 0 & 0 & 0 \\
0 & I_{\mathcal{H}_{1}} & 0 & 0 & 0 & 0 \\
0 & 0 & I_{\mathcal{H}^{\prime}} & 0 & 0 & 0 \\
0 & 0 & 0 & 0_{\mathcal{H}^{\prime}} & 0 & 0 \\
0 & 0 & 0 & 0 & 0_{\mathcal{H}_{2}} & 0 \\
0 & 0 & 0 & 0 & 0 & 0_{\mathcal{H}_{3}}
\end{array}\right) \text { and } Q=\left(\begin{array}{cccccc}
0_{\mathcal{H}_{0}} & 0 & 0 & 0 & 0 & 0 \\
0 & I_{\mathcal{H}_{1}} & 0 & 0 & 0 & 0 \\
0 & 0 & S & X & 0 & 0 \\
0 & 0 & X & I_{\mathcal{H}^{\prime}}-S & 0 & 0 \\
0 & 0 & 0 & 0 & I_{\mathcal{H}_{2}} & 0 \\
0 & 0 & 0 & 0 & 0 & 0_{\mathcal{H}_{3}}
\end{array}\right),
$$

where $X=\sqrt{S\left(I_{\mathcal{H}^{\prime}}-S\right)} \in \mathcal{L}\left(\mathcal{H}^{\prime}\right)$.

Proof. Again, the representation results follow from standard matrix computations.

The following question happens frequently in many concrete problems in operator theory.

Question 2.2. When is $\mathcal{P}+\mathcal{Q}$ closed in $\mathcal{H}$, where $\mathcal{P}=\operatorname{ran} P$ and $\mathcal{Q}=\operatorname{ran} Q$ ?

Note we have:

$$
\begin{gathered}
\mathcal{P}=\left\{\left(\begin{array}{c}
x_{0} \\
x_{1} \\
x^{\prime} \\
0 \\
0 \\
0
\end{array}\right): x_{0} \in \mathcal{H}_{0}, x_{1} \in \mathcal{H}_{1}, x^{\prime} \in \mathcal{H}^{\prime}\right\} \text { and } \\
\mathcal{Q}=\left\{\left(\begin{array}{c}
0 \\
x_{1} \\
S x^{\prime}+X y^{\prime} \\
X x^{\prime}+\left(I_{\mathcal{H}^{\prime}}-S\right) y^{\prime} \\
x_{2} \\
0
\end{array}\right): x_{1} \in \mathcal{H}_{1}, x^{\prime}, y^{\prime} \in \mathcal{H}^{\prime}, x_{2} \in \mathcal{H}_{2}\right\} .
\end{gathered}
$$


Therefore,

$$
\mathcal{P}+\mathcal{Q}=\left\{\left(\begin{array}{c}
x_{0} \\
x_{1} \\
x^{\prime} \\
z \\
x_{2} \\
0
\end{array}\right): x_{0} \in \mathcal{H}_{0}, x_{1} \in \mathcal{H}_{1}, x^{\prime} \in \mathcal{H}^{\prime}, z \in \operatorname{ran} X+\operatorname{ran}\left(I_{\mathcal{H}^{\prime}}-S\right), x_{2} \in \mathcal{H}_{2}\right\} .
$$

This implies that $\mathcal{P}+\mathcal{Q}$ is closed if and only if $\operatorname{ran} X+\operatorname{ran}\left(I_{\mathcal{H}^{\prime}}-S\right)$ is closed. Since $X=\sqrt{S\left(I_{\mathcal{H}^{\prime}}-S\right)}$, we have that $\operatorname{ran} X \subseteq \operatorname{ran}\left(I_{\mathcal{H}^{\prime}}-S\right)^{\frac{1}{2}}$. Moreover, by the spectral theorem for the positive contraction $S$, one sees that $\sqrt{S}+\sqrt{I_{\mathcal{H}^{\prime}}-S}$ is invertible on $\mathcal{H}^{\prime}$. This implies that $\operatorname{ran} X+\operatorname{ran}\left(I_{\mathcal{H}^{\prime}}-S\right) \supseteq \operatorname{ran}\left(I_{\mathcal{H}^{\prime}}-S\right)^{\frac{1}{2}}$. Therefore, $\operatorname{ran} X+\operatorname{ran}\left(I_{\mathcal{H}^{\prime}}-S\right)$ is closed if and only if $\operatorname{ran}\left(I_{\mathcal{H}^{\prime}}-S\right)^{\frac{1}{2}}$ is closed. Since 1 is not in the point spectrum of $S$, it follows from the spectral theorem that $\operatorname{ran}\left(I_{\mathcal{H}^{\prime}}-S\right)^{\frac{1}{2}}$ is closed if and only if 1 is not in the spectrum of $S$. Hence we have the following result.

Theorem 2.3. For two projections $P$ and $Q$ on the Hilbert space $\mathcal{H}$ with $\mathcal{P}=$ ranP and $\mathcal{Q}=$ ran $Q$, the linear span $\mathcal{P}+\mathcal{Q}$ is closed if and only if $1 \notin \sigma(S)$, or equivalently, $\sigma(P Q P) \cap(\varepsilon, 1)=\phi$ for some $0<\varepsilon<1$, where $S$ is the same as in Theorem 2.1 . Moreover, in the case that $\mathcal{R}=\mathcal{P}+\mathcal{Q}$ is closed, the projection $R$ onto $\mathcal{R}$ has the form

$$
R=\left(\begin{array}{cccccc}
I_{\mathcal{H}_{0}} & 0 & 0 & 0 & 0 & 0 \\
0 & I_{\mathcal{H}_{1}} & 0 & 0 & 0 & 0 \\
0 & 0 & I_{\mathcal{H}^{\prime}} & 0 & 0 & 0 \\
0 & 0 & 0 & I_{\mathcal{H}^{\prime}} & 0 & 0 \\
0 & 0 & 0 & 0 & I_{\mathcal{H}_{2}} & 0 \\
0 & 0 & 0 & 0 & 0 & 0_{\mathcal{H}_{3}}
\end{array}\right)
$$

Proof. Only the last statement remains to be proved and that follows from the fact that $1 \notin \sigma(S)$ if and only if $0 \notin \sigma\left(I_{\mathcal{H}^{\prime}}-S\right)$, which implies $\operatorname{ran}\left(I_{\mathcal{H}^{\prime}}-S\right)=\mathcal{H}^{\prime}$.

A nearly immediate consequence of the representation theorem is the following characterization of the $C^{*}$-algebra $\mathcal{A}(P, Q, I)$ generated by projections $P, Q$ and the identity operator on the Hilbert space $\mathcal{H}$. This is usually attributed to Dixmier [9].

Theorem 2.4. Let $P$ and $Q$ be the projections onto the subspaces $\mathcal{P}$ and $\mathcal{Q}$ of the Hilbert space $\mathcal{H}$, respectively. If $\mathcal{P} \cap \mathcal{Q}=\mathcal{P}^{\perp} \cap \mathcal{Q}=\mathcal{P} \cap \mathcal{Q}^{\perp}=\mathcal{P}^{\perp} \cap \mathcal{Q}^{\perp}=\{0\}$, then $\mathcal{A}(P, Q, I)$ is $*$-algebaically isomorphic to a ${ }^{*}$-subalgebra $\mathcal{C}$ of $M_{2}(C(M))$, where $M=\sigma(P Q P), M_{2}(C(M))$ denotes the algebra of two by two matrices with entries in $C(M)$ and

$$
\mathcal{C}=\left\{\left(\begin{array}{ll}
\phi_{11} & \phi_{12} \\
\phi_{21} & \phi_{22}
\end{array}\right) \in M_{2}(C(M)): \phi_{12}(i)=\phi_{21}(i)=0 \text {, if } i=0,1 \text { and } i \in M\right\} .
$$

Proof. Applying the spectral theorem to the operators $I_{\mathcal{P}}$ and $S$, one obtains the correspondence from which the result follows:

$$
\begin{gathered}
P=\left(\begin{array}{cc}
I_{\mathcal{P}} & 0 \\
0 & 0
\end{array}\right) \sim\left(\begin{array}{ll}
1 & 0 \\
0 & 0
\end{array}\right) \in M_{2}(C(M)) \text { and } \\
Q=\left(\begin{array}{cc}
S & \sqrt{S(1-S)} \\
\sqrt{S(1-S)} & 1-S
\end{array}\right) \sim\left(\begin{array}{cc}
\chi & \sqrt{\chi(1-\chi)} \\
\sqrt{\chi(1-\chi)} & 1-\chi
\end{array}\right) \in M_{2}(C(M)),
\end{gathered}
$$


where 1 and $\chi$ denote the functions on $M$ defined by $1(x)=1$ and $\chi(x)=x$ for $x \in M$. The fact that the functions $\phi_{12}$ and $\phi_{21}$ in the definition of $\mathcal{C}$ vanish at $0,1 \in M$ follows from the fact that the function $\sqrt{\chi(1-\chi)}$ does.

We now use the characterization of the $C^{*}$-algebra generated by two projections to get our first result on the essential normality of the projection onto the linear span when it is closed.

Theorem 2.5. For two projections $P$ and $Q$ on the Hilbert space $\mathcal{H}$, if $\mathcal{R}=$ ranP+ ranQ is closed, then the $C^{*}$-algebra $\mathcal{A}\left(P, Q, I_{\mathcal{H}}\right)$ generated by $P, Q$ and the identity operator $I_{\mathcal{H}}$ contains the projection $R$ onto the subspace $\mathcal{R}$.

Proof. Using a direct matrix computation, one sees that the operator $P+(I-P) Q(I-$ $P)$, which is in the $C^{*}$-algebra $\mathcal{A}(P, Q, I)$, has the form

$$
P+(I-P) Q(I-P)=\left(\begin{array}{cccccc}
I_{\mathcal{H}_{0}} & 0 & 0 & 0 & 0 & 0 \\
0 & I_{\mathcal{H}_{1}} & 0 & 0 & 0 & 0 \\
0 & 0 & I_{\mathcal{H}^{\prime}} & 0 & 0 & 0 \\
0 & 0 & 0 & I_{\mathcal{H}^{\prime}}-S & 0 & 0 \\
0 & 0 & 0 & 0 & I_{\mathcal{H}_{2}} & 0 \\
0 & 0 & 0 & 0 & 0 & 0_{\mathcal{H}_{3}}
\end{array}\right)
$$

This implies that $\sigma(P+(I-P) Q(I-P)) \subseteq\{0\} \cup[\varepsilon, 1]$ for some $0<\varepsilon<1$. Since $[\varepsilon, 1] \cap \sigma(P+(I-P) Q(I-P))$ is an open and closed subset of the spectrum $\sigma(P+$ $(I-P) Q(I-P))$, it follows from the spectral theorem that the spectral projection $\mathbf{1}_{[\varepsilon, 1]}(P+(I-P) Q(I-P))$ is in $\mathcal{A}(P, Q, I)$, which leads to the desired result since $\mathbf{1}_{[\varepsilon, 1]}(P+(I-P) Q(I-P))=R$.

We now relate the representation result to a question in the context of Arveson's conjecture. We will provide a more precise statement in Section 4.

Theorem 2.6. Suppose $\sigma: C(M) \rightarrow \mathcal{L}(\mathcal{H})$ is a $*$-homomorphism for some compact metric space $M$, and $P, Q$ are projections on the Hilbert space $\mathcal{H}$ such that the commutators $[\sigma(\varphi), P] \in \mathcal{K}$ and $[\sigma(\varphi), Q] \in \mathcal{K}$ for $\varphi \in C(M)$, where $\mathcal{K}$ denotes the ideal of compact operators on $\mathcal{H}$. If $\mathcal{R}=$ ran $P+$ ran $Q$ is closed and $R$ is the projection onto $\mathcal{R}$, then $[\sigma(\varphi), R] \in \mathcal{K}$ for $\varphi \in C(M)$.

Proof. Using an elementary $C^{*}$-algebra argument, one shows that $[\sigma(\varphi), T] \in \mathcal{K}$ for any operator $T \in \mathcal{A}(P, Q, I)$. Combining this fact with Theorem 2.5, one obtains the desired result.

Corollary 2.7. With the same hypotheses, the projection $\widetilde{R}$ onto $\mathcal{P} \cap \mathcal{Q}$ essentially commutes with the range of $\sigma$.

Proof. This is an immediate consequence of Theorem 1 in [11] and the exact sequence

$$
0 \rightarrow \widetilde{R} \stackrel{i}{\rightarrow} \mathcal{P} \oplus \mathcal{Q} \stackrel{j}{\rightarrow} \mathcal{R} \rightarrow 0
$$

where $i(r)=(r,-r)$, and $j(p, q)=p+q$ for $r \in \widetilde{R}, p \in \mathcal{P}, q \in \mathcal{Q}$.

Remark 2.8. In both the theorem and corollary, $C(M)$ can be replaced by any $C^{*}$ subalgebra of $\mathscr{L}(\mathcal{H})$. 
Remark 2.9. These results are related to a theorem of Arveson [5, Theorem 4.4] and the more recent work of Kennedy [19] in which essential normality is replaced by $p$-essential normality, where the commutators are assumed to be in the Schatten $p$-class for $1 \leq$ $p<\infty$. If one examines the proof of Theorem 2.5 more closely, the preceding arguments can be refined to obtain analogous results for $p$-essential normality. Basically, this is true because the functional calculus which yields the spectral projection $\mathbf{1}_{[\varepsilon, 1]}(P+(I-$ $P) Q(I-P))$ can be approximated on a neighborhood of the spectrum with analytic functions.

We can extend these results somewhat using the following reduction which is essentially algebraic.

Theorem 2.10. Suppose $\mathcal{P}$ and $\mathcal{Q}$ are subspaces of the Hilbert space $\mathcal{H}$ and $\mathcal{R}^{\sharp}$ is a subspace of $\mathcal{P} \cap \mathcal{Q}$. Then $\mathcal{P}+\mathcal{Q}$ is closed if and only if $\mathcal{P} / \mathcal{R}^{\sharp}+\mathcal{Q} / \mathcal{R}^{\sharp}$ is closed in $\mathcal{H} / \mathcal{R}^{\sharp}$.

Proof. It follows from the fact that $\mathcal{P} / \mathcal{R}^{\sharp}+\mathcal{Q} / \mathcal{R}^{\sharp}=(\mathcal{P}+\mathcal{Q}) / \mathcal{R}^{\sharp}$ and the fact that for any linear manifold $\mathcal{L}$ containing $\mathcal{R}^{\sharp}, \mathcal{L} / \mathcal{R}^{\sharp}$ is closed if and only if $\mathcal{L}$ is closed.

Corollary 2.11. With the same hypotheses, the closeness of $\mathcal{P} / \mathcal{R}^{\sharp}+\mathcal{Q} / \mathcal{R}^{\sharp}$ is equivalent to the closeness of $\mathcal{P} /(\mathcal{P} \cap \mathcal{Q})+\mathcal{Q} /(\mathcal{P} \cap \mathcal{Q})$.

Proof. Both of these statements are equivalent to $\mathcal{P}+\mathcal{Q}$ being closed in $\mathcal{H}$.

\section{Essential Span of Subspaces}

The following question and corresponding result are important for considering the notion of essential span in this section.

Question 3.1. When do two projections $P$ and $Q$ on a Hilbert space $\mathcal{H}$ almost commute; that is, when is $[P, Q] \in \mathcal{K}(\mathcal{H})$ ?

Using the representation theorem for $P$ and $Q$ above, we see that $[P, Q] \in \mathcal{K}$ if and only if $X \in \mathcal{K}$ and so we have the following result.

Theorem 3.2. For projections $P$ and $Q$ onto subspaces $\mathcal{P}$ and $\mathcal{Q}$, respectively, on a Hilbert space $\mathcal{H},[P, Q] \in \mathcal{K}$ if and only if $\sigma_{e}(S) \subset\{0,1\}$. Moreover, $P Q \in \mathcal{K}$ if and only if $S \in \mathcal{K}$ and $\operatorname{dim} \mathcal{P} \cap \mathcal{Q}<\infty$ in the representation appearing in Theorem 2.1.

Proof. The proof follows from a matrix calculation in the above representation theorem which shows that $[P, Q] \in \mathcal{K}$ if and only if $X=\sqrt{S\left(I_{\mathcal{H}^{\prime}}-S\right)}$ is compact. For $P Q \in \mathcal{K}$, it is necessary and sufficient for $S$ and $I_{\mathcal{H}_{1}}$ to be compact.

If $P$ and $Q$ are projections on the Hilbert space $\mathcal{H}$, then another notion of the span of the ranges of $P$ and $Q$ is relevant when considering questions of essential normality, which involves the images of $P$ and $Q$ in the Calkin algebra. If 0 is an isolated point in the essential spectrum, $\sigma_{e}(P+Q)$, of $P+Q$, or $0 \notin \sigma_{e}(P+Q)$, then the image in the Calkin algebra of the spectral projection, $P \vee_{e} Q$, for $[\varepsilon, \infty]$, where $(0, \varepsilon) \cap \sigma_{e}(P+Q)=\phi$, can be thought of as the "essential span" of $\operatorname{ran} P$ and $\operatorname{ran} Q$. (Note that the image of this spectral projection in the Calkin algebra does not depend on $\varepsilon$ whenever $(0, \varepsilon) \cap \sigma_{e}(P+Q)=\phi$.) One result related to this notion is the following. 
Theorem 3.3. If $[P, Q] \in \mathcal{K}$, then 0 is isolated in $\sigma_{e}(P+Q)$. Moreover, if $P$ and $Q$ almost commute with a $C^{*}$-algebra $\mathfrak{A}$, then so does any projection on $\mathcal{H}$ with the image $P \bigvee_{e} Q$ in the Calkin algebra.

Proof. Considering the standard model for two projection in Section 2, one sees that $[P, Q] \in \mathcal{K}$ implies that $X$ is compact and $\sigma_{e}(P+Q) \subseteq\{0,1,2\}$. This implies that $[\varepsilon, 2]$ is an open and closed subset of $\sigma(P+Q)$ for some $0<\varepsilon<1$ and hence $\mathbf{1}_{[\varepsilon, 2]}(P+Q)$ is in $\mathcal{A}(P, Q, I)$, where $\mathcal{A}(P, Q, I)$ is the $C^{*}$-algebra generated by $P, Q$ and the identity operator $I$. Therefore, its image in the Calkin algebra, $P \bigvee_{e} Q$, commutes with the image of $\mathfrak{A}$, which completes the proof.

One thing one needs to be clear on is that the image of $P \bigvee Q$ in the Calkin algebra and $P \bigvee_{e} Q$ are not necessarily the same. Consider, for example, the subspaces $\mathcal{P}=$ $\overline{\operatorname{span}}\left\{e_{n} \oplus 0: n \in \mathbb{N}\right\}$ in $\ell^{2} \oplus \ell^{2}$ and $\mathcal{Q}=\overline{\operatorname{span}}\left\{e_{n} \oplus \frac{1}{n} e_{n}: n \in \mathbb{N}\right\}$. These subspaces have the images of $\pi(P \bigvee Q)$ and $P \bigvee_{e} Q$ in the Calkin algebra which are the images of the projections onto $\ell^{2} \oplus \ell^{2}$ and $\ell^{2} \oplus(0)$, respectively. Note that in this case $\mathcal{P}+\mathcal{Q}$ is not closed, which is the key as the following result shows.

Theorem 3.4. Let $P, Q$ be the projections onto the subspaces $\mathcal{P}$ and $\mathcal{Q}$ of a Hilbert space $\mathcal{H}$, respectively. Then $\mathcal{P}+\mathcal{Q}$ is closed if and only if $\pi(P \bigvee Q)=P \bigvee_{e} Q$.

Proof. We first suppose that $\mathcal{P}+\mathcal{Q}$ is closed. Using the notation in Theorem 2.1, we have that

$$
P+Q=\left(\begin{array}{cccccc}
I_{\mathcal{H}_{0}} & 0 & 0 & 0 & 0 & 0 \\
0 & 2 I_{\mathcal{H}_{1}} & 0 & 0 & 0 & 0 \\
0 & 0 & I_{\mathcal{H}^{\prime}}+S & \sqrt{S\left(I_{\mathcal{H}^{\prime}}-S\right)} & 0 & 0 \\
0 & 0 & \sqrt{S\left(I_{\mathcal{H}^{\prime}}-S\right)} & I_{\mathcal{H}^{\prime}}-S & 0 & 0 \\
0 & 0 & 0 & 0 & I_{\mathcal{H}_{2}} & 0 \\
0 & 0 & 0 & 0 & 0 & 0_{\mathcal{H}_{3}}
\end{array}\right)
$$

By Theorem 2.3 we know that $1 \notin \sigma(S)$ when $\mathcal{P}+\mathcal{Q}$ is closed. Applying the spectral theorem to the operator $S$, one obtains that 0 is isolated in $\sigma(P+Q)$. This implies that the notion $P \bigvee_{e} Q$ makes sense and, in fact, it is the image in the Calkin algebra of the projection onto $\operatorname{ran}(P+Q)$. Moreover, by the above representation of $P+Q$, one sees that

$$
\operatorname{ran}(P+Q)=\mathcal{H}_{0} \oplus \mathcal{H}_{1} \oplus \mathcal{H}^{\prime} \oplus \mathcal{H}^{\prime} \oplus \mathcal{H}_{2}=\mathcal{P}+\mathcal{Q}
$$

It follows that $P \bigvee_{e} Q$ is the image of the projection onto $\mathcal{P}+\mathcal{Q}$.

On the other hand, in case that $\pi(P \bigvee Q)=P \bigvee_{e} Q$, there exists $0<\varepsilon<1$ such that $(0, \varepsilon) \cap \sigma_{e}(P+Q)=\phi$ and $P \bigvee Q-\mathbf{1}_{[\varepsilon, \infty]}(P+Q)$ is a finite dimensional projection. Applying the spectral theorem for $S$ to the matrix representation of $P+Q$, one sees that the spectral projection of $S$ for $(1-\varepsilon, 1)$ is also a finite dimensional projection. Combing this fact with that 1 is not in the point spectrum of $S$, we have that $1 \notin \sigma(S)$, which leads to the desired result using Theorem 2.3.

While it seems inconceivable that $[p]+[q]$ is always closed for polynomials $p$ and $q$ in $\mathbb{C}\left[z_{1}, \cdots, z_{n}\right]$; here $[\cdot]$ denotes the closure in the Hardy, Bergman or Drury-Arveson modules on the unit ball, it seems quite possible that the projections onto $[p]$ and $[q]$ always almost commute. One thing making the answering of such a question difficult is the fact that $[p] \cap[q]$ is always large containing $[p q]$. One possible way to circumvent 
this problem might be to consider the quotient modules $[p]^{\perp}$ and $[q]^{\perp}$. We'll have something more to say about them in the next section.

Another possibility to handle the fact that $[p] \cap[q]$ is large might be to use Theorem 2.10 to reduce the matter to $[p] /([p] \cap[q])$ and $[q] /([p] \cap[q])$. In this case, $[p] /([p] \cap[q])$ and $[q] /([p] \cap[q])$ are semi-invariant modules. We will obtain a result using this approach in the following section.

\section{Locality of Essentially Normal Projection}

Let $M$ be a compact metric space and $\sigma: C(M) \rightarrow \mathscr{L}(\mathcal{H})$ be a $*$-homomorphism for a Hilbert space $\mathcal{H}$. We say that a projection $P$ on $\mathcal{H}$ is essentially normal relative to $\sigma$ if $[\sigma(\varphi), P] \in \mathcal{K}$ for any $\varphi \in C(M)$. This implies that the map $\sigma_{P}: \varphi \rightarrow \pi(P \sigma(\varphi) P) \in$ $\mathscr{Q}(\mathcal{H})$ into the Calkin algebra $\mathscr{Q}(\mathcal{H})=\mathscr{L}(\mathcal{H}) / \mathcal{K}(\mathcal{H})$ is a $*$-homomorphism. Hence, there exists a compact subset $M_{P}$ of $M$ such that the following diagram commutes:

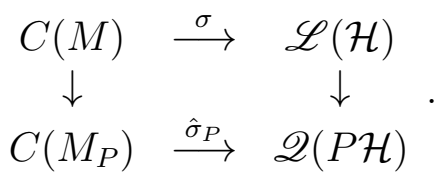

Here the vertical arrow on the left is defined by restriction; that is, $\left.\varphi \rightarrow \varphi\right|_{M_{P}}$, and the one on the right is the compression to ran $P$ followed by the map onto the Calkin algebra. Therefore, using [7], one knows that $(\sigma, P)$ defines an element $[\sigma, P] \in K_{1}\left(M_{P}\right)$. An interesting question concerns the relation of elements $[\sigma, P]$ and $[\sigma, Q]$ for two essentially normal projections $P$ and $Q$ relative to $\sigma$.

Now this relationship can't be too simple. In particular, consider the representation $\tau$ of $C\left(\operatorname{clos} \mathbb{B}^{n}\right)$ in $L^{2}\left(\mathbb{B}^{n}\right)$ and the projection $P$ onto the Bergman space $L_{a}^{2}\left(\mathbb{B}^{n}\right)$. For $p \in \mathbb{C}\left[z_{1}, \cdots, z_{n}\right]$, one knows [13] that the projection $Q_{p}$ of $L^{2}\left(\mathbb{B}^{n}\right)$ onto the closure $[p]$ of the ideal $(p)$ in $\mathbb{C}\left[z_{1}, \cdots, z_{n}\right]$ generated by $p$ is essentially normal; that is, $\left[\tau(\varphi), Q_{p}\right] \in \mathcal{K}$ for $\varphi \in C\left(\operatorname{clos} \mathbb{B}^{n}\right)$. Further, we have that $R_{p}=P-Q_{p}$ is also essentially normal and $M_{\left[\tau, R_{p}\right]} \subseteq Z(p) \cap \partial \mathbb{B}^{n}$, where $Z(p)$ is the zero variety of the polynomial $p$. It follows that the image of $\left[\tau, R_{p}\right] \in K_{1}\left(\partial \mathbb{B}^{n}\right)$ is zero since $Z(p) \cap \partial \mathbb{B}^{n}$ is a proper subset of $\partial \mathbb{B}^{n}$. Therefore, one has $[\tau, P]=\left[\tau, Q_{p}\right]$ for every polynomial $p \in \mathbb{C}\left[z_{1}, \cdots, z_{n}\right]$. Hence, there is a great variety of essentially normal projections defining the same element in $K_{1}\left(\partial \mathbb{B}^{n}\right)$.

However, we do have a result for what happens at the opposite extreme.

Theorem 4.1. Suppose that $P$ and $Q$ are essentially normal projections on the Hilbert space $\mathcal{H}$ for the $*$-homomorphism $\sigma: C(M) \rightarrow \mathscr{L}(\mathcal{H})$ for some compact space $M$. If $M_{P} \cap M_{Q}=\phi$, then $P Q \in \mathcal{K}$.

Proof. By the assumption that $P$ and $Q$ are essentially normal relative to $\sigma$, one sees that the operator $P Q$ almost intertwines the two representations $\left.\sigma\right|_{C\left(M_{P}\right)}$ and $\left.\sigma\right|_{C\left(M_{Q}\right)}$; that is, one has that $P \sigma(\varphi) P(P Q)-(P Q) Q \sigma(\varphi) Q \in \mathcal{K}$ for $\varphi \in C(M)$. Thus, in the Calkin algebra, if $\varphi \in C(M)$ satisfies $\left.\varphi\right|_{M_{P}} \equiv 1$ and $\left.\varphi\right|_{M_{Q}} \equiv 0$, we obtain that $\pi(Q \sigma(\varphi) Q) \pi(P Q)=\pi(P Q) \pi(P \sigma(\varphi) P)$. But, $\pi(Q \sigma(\varphi) Q)=0$ and $\pi(P \sigma(\varphi) P)=$ $\pi(P)$, this means that $\pi(P Q P)=0$, which implies $P Q \in \mathcal{K}$ and completes the proof.

We can use this theorem to obtain a partial result concerning the relation of the projections onto $[p]$ and $[q]$ for $p, q \in \mathbb{C}\left[z_{1}, \cdots, z_{n}\right]$. 
Corollary 4.2. For two polynomials $p, q \in \mathbb{C}\left[z_{1}, \cdots, z_{n}\right]$, let $P$ and $Q$ be the projections onto the submodule $\mathcal{P}=[p], \mathcal{Q}=[q]$ on $L_{a}^{2}\left(\mathbb{B}^{n}\right)$, respectively. If $p, q$ satisfy $Z(p) \cap Z(q) \cap$ $\partial \mathbb{B}^{n}=\phi$, then we have that $[P, Q] \in \mathcal{K}$.

Proof. Note that $I-P, I-Q$ are the projections onto the quotient modules $\mathcal{P}^{\perp}$ and $\mathcal{Q}^{\perp}$, respectively. Using the notation in the above, by [13] we know that $M_{I-P} \subseteq Z(p) \cap \partial \mathbb{B}^{n}$ and $M_{I-Q} \subseteq Z(q) \cap \partial \mathbb{B}^{n}$. It follows from the hypothesis that $M_{I-P} \cap M_{I-Q}=\phi$. By Theorem 4.1 we have that $(I-P)(I-Q)$ and $(I-Q)(I-P)$ are compact. Therefore, one sees that $[P, Q] \in \mathcal{K}$, which completes the proof.

We can extend this result using Theorem 2.10 as follows.

Corollary 4.3. For polynomials $p, q, r \in \mathbb{C}\left[z_{1}, \cdots, z_{n}\right]$ with $Z(p) \cap Z(q) \cap \partial \mathbb{B}^{n}=\phi$, let $\mathcal{P}=[p r]$ and $\mathcal{Q}=[q r]$ be the submodules in $L_{a}^{2}\left(\mathbb{B}^{n}\right)$. Then one has $[P, Q] \in \mathcal{K}$, where $P, Q$ are the projections onto $\mathcal{P}$ and $\mathcal{Q}$, respectively.

Proof. One can generalize the argument in [13] to show that

$$
M_{[p r]^{\perp} /[r]^{\perp}} \subseteq Z(p) \cap \partial \mathbb{B}^{n} \text { and } M_{[q r]^{\perp} /[r]^{\perp}} \subseteq Z(q) \cap \partial \mathbb{B}^{n} .
$$

By Theorem 4.1, this implies that $(R-P)(R-Q)$ and $(R-Q)(R-P)$ are compact, where $R$ is the projection onto the submodule $[r]$. This means that $[P, Q]=[R-P, R-$ $Q] \in \mathcal{K}$, which completes the proof.

Another example of the application of the notion of the locality of essentially normal projection is the following result which is more or less the opposite situation of the previous theorem.

Theorem 4.4. Assume that $\sigma: C(M) \rightarrow \mathscr{Q}(\mathcal{H})$ is a*-homomorphism on the Hilbert space $\mathcal{H}$ for a compact metric space $M$, and $P$ and $Q$ are two essentially normal projections such that $\mathcal{P} \cap \mathcal{Q}^{\perp}=\mathcal{P}^{\perp} \cap \mathcal{Q}=\{0\}$, where $\mathcal{P}=\operatorname{ranP}$ and $\mathcal{Q}=$ ran $Q$. If $\mathcal{P}+\mathcal{Q}^{\perp}$ is closed, then $M_{P}=M_{Q}$ and $\left[\hat{\sigma}_{P}\right]=\left[\hat{\sigma}_{Q}\right] \in K_{1}\left(M_{P}\right)$.

Proof. In the representation theorem for $P, Q$, the spaces $\mathcal{H}_{0}$ and $\mathcal{H}_{2}$ are $\{0\}$ by assumption and we can write $\mathcal{P}=\mathcal{H}_{1} \oplus \mathcal{P}^{\prime}$ and $\mathcal{Q}=\mathcal{H}_{1} \oplus \mathcal{Q}^{\prime}$ corresponding to $P^{\prime}=P-I_{\mathcal{H}_{1}}$ and $Q^{\prime}=Q-I_{\mathcal{H}_{1}}$. As in the proof of Theorem 4.1, the image $\pi\left(P^{\prime} Q^{\prime}\right)$ of $P^{\prime} Q^{\prime}$ in the Calkin algebra intertwines the operators $\pi\left(P^{\prime} \sigma(\varphi) P^{\prime}\right)$ and $\pi\left(Q^{\prime} \sigma(\varphi) Q^{\prime}\right)$. Using Theorem 2.3 and the assumption $\mathcal{P}+\mathcal{Q}^{\perp}$ is closed, we have $0 \notin \sigma(S)=\sigma\left(P^{\prime} Q^{\prime} P^{\prime}\right)$. Combining this with the fact $\operatorname{ker} P^{\prime} Q^{\prime}=\mathcal{P}^{\prime \perp} \cap \mathcal{Q}^{\prime}=\{0\}$, one sees that $P^{\prime} Q^{\prime}: \mathcal{Q}^{\prime} \rightarrow \mathcal{P}^{\prime}$ is invertible. Therefore, using the polar decomposition in the Calkin algebra, one sees that $M_{P}=M_{Q}$ and that the $K_{1}$ elements are equal.

There would seem to be a limit to what can be concluded about the $K_{1}$ element. If $k \in K_{1}\left(M_{P}\right)$ for some essentially normal projection $P$ on the Hilbert space $\mathcal{H}$ with a $*-$ homomorphism $\sigma: C(M) \rightarrow \mathscr{L}(\mathcal{H})$, then there exists an essentially normal projection $Q \leq P$ such that $[\sigma, Q]=k \in K_{1}\left(M_{P}\right)$

\section{REFERENCES}

[1] W. B. Arveson, Subalgebras of $C^{*}$-algebras III: Multivariate operator theory, Acta Math., 181(1998), 159-228.

[2] W. B. Arveson, The curvature invariant of a Hilbert module over $\mathbb{C}\left[z_{1}, \ldots, z_{d}\right]$, J. reine angew. Math., 522(2000), 173-236. 
[3] W. B. Arveson, The Dirac operator of a commuting d-tuple, J. Funct. Anal., 189 (2002), 53-79.

[4] W. B. Arveson, p-Summable commutators in dimension d, J. Oper. Theory, 54(2005), 101-117.

[5] W. B. Arveson, Quotients of standard Hilbert modules, Trans. Amer. Math. Soc., 359(2007), 6027-6055.

[6] A. Brown, The unitary equivalence of binormal operators, Amer. J. Math., 76(1954), 414-434.

[7] L.G. Brown, R.G. Douglas and P.A. Fillmore, Extensions of $C^{*}$-algebras and $K$-homology, Ann. Math., 105 (1977), 265-324.

[8] C. Berger and B. Shaw, Selfcommutators of multicyclic hyponormal operators are always trace class, Bull. Amer. Math. Soc., 79(1973), 1193-1199.

[9] J. Dixmier, Position relative de deux variétes linéaires fermées dans un espace de Hilbert, Rev. Sci., 86(1948), 387-399.

[10] R. G. Douglas, A new kind of index theorem, Analysis, Geometry and Topology of Elliptic Operators (Roskilde, Denmark, 2005), 369-382, World Sci. Publishing, 2006.

[11] R. G. Douglas, Essentially reductive Hilbert modules, J. Oper. Theory, 55(2006), 117-133.

[12] R. G. Douglas and J. Sarkar, Essentially reductive weighted shift Hilbert modules, J. Oper. Theory, 65(2011), 101-133.

[13] R. G. Douglas and K. Wang, A harmonic analysis approach to essential normality of principal submodules, J. Funct. Anal., 261(2011), 3155-3180.

[14] J. Eschmeier, Essential normality of homogeneous submodules, Int. Eq. Oper. Ther., 69(2011), 171-182.

[15] K. Guo, Defect operators for submodules of $H_{d}^{2}$, J. reine angew. Math., 573(2004), 181-209.

[16] K. Guo and K. Wang, Essentially normal Hilbert modules and K-homology II: Quasihomogeneous Hilbert modules over the two dimensional unit ball, J. Ramanujan Math. Soc., 22 (2007), 259-281.

[17] K. Guo and K. Wang, Essentially normal Hilbert modules and K-homology, Math. Ann., 340(2008), 907-934.

[18] P. R. Halmos, Two subspaces, Trans. Amer. Math. Soc., 144(1969), 381-389.

[19] M.Kennedy, Essential normality and the decomposability of homogeneous submodules, arXiv:1202.1797.

[20] O. Shalit, Stable polynomial division and essential normality of graded Hilbert modules, J. London Math. Soc., 83(2011), 273-289.

Department of Mathematics, Texas A\&M University, College Station, TX 77843, USA

E-mail address: rdouglas@math.tamu.edu

School of Mathematical Sciences, Fudan University, Shanghai, 200433, P. R. China

E-mail address: kwang@fudan.edu.cn 